\title{
Modelling of e-Cloud Induced Coherent Tuneshifts Using POSINST: Simulation of April 2007 Measurements at Cesr
}

\author{
Marco Venturini* \\ Lawrence Berkeley National Laboratory, University of California, Berkeley, California, 94720
}

(Dated: April 15, 2009)

PACS numbers:

\section{INTRODUCTION}

We model the interaction between a beam and electron cloud (EC) as taking place in localized stations along the accelerator at $s=s_{i}, i=0,1,2, \ldots, n_{s}$. A beam particle with charge $q$ interacting with the electron cloud at each station receives a, say, vertical kick

$$
\Delta y^{\prime}=\Delta s\left(\frac{d^{2} y}{d s^{2}}\right)_{E C}=\frac{q \Delta s}{v_{0} p_{0}} E_{y}\left(x, y, s=s_{i} ; t\right) \simeq \frac{q \Delta s}{E_{0}} E_{y}\left(x, y, s=s_{i} ; t\right),
$$

where $v_{0}, p_{0}$, and $E_{0}$ are the beam nominal velocity, momentum and energy, and $E_{y}\left(x, y, s=s_{i} ; t\right)$ the electric field due to the electrons present at station $s_{i}$ at time $t$ of the beam particle passage; $\Delta s$ is the length of the physical section of the machine $\left[s_{i}-\Delta s_{i} / 2, s_{i}+\Delta s_{i} / 2\right]$ hosting the EC station. A similar equation will hold for the horizontal kick.

Suppose that the particle belongs to a beam consisting of $k=1,2,3, \ldots n_{b}$ bunches, each bunch being described by a density in configuration space $\rho_{k}(x, y, \tau)=\rho_{\perp, k}(x, y) \rho_{\|, k}(\tau)$, where $\tau=-z / v_{0}$ is the time of flight relative to the reference particle. For the longitudinal density we also use the notation $\rho_{z}(z)=\rho_{\|}\left(\tau=-z / v_{0}\right) ; \rho_{\perp}(x, y)$ and $\rho_{z}(z)$ are assumed to be normalized to unity.

The kick received by the centroid of a beam slice located at $z$ is then

$$
\Delta\left\langle y^{\prime}(z)\right\rangle_{\perp}=\frac{q \Delta s_{i}}{E_{0}} \int d x d y E_{y}\left(x, y, s=s_{i} ; k, z\right) \rho_{\perp, k}(x, y)
$$

where $\left\langle y^{\prime}\right\rangle_{\perp}=\int d x d y y^{\prime} \rho_{\perp}(x, y)$. In the notation of the electric field $E_{y}$ in $(2)$ we have replaced the time of particle passage $t$ with the equivalent pair of numbers $k$ and $z$. Similarly, after averaging over the entire bunch density, the kick to the centroid of bunch $k$ reads

$$
\Delta\left\langle y_{k}^{\prime}\right\rangle=\frac{q \Delta s_{i}}{E_{0}} \int d x d y d z E_{y}\left(x, y, s=s_{i} ; k, z\right) \rho_{k}(x, y, z)
$$

where $\left\langle y^{\prime}\right\rangle=\int d z \rho_{z}(z)\left\langle y^{\prime}\right\rangle_{\perp}$ represents the averaging over the $3 \mathrm{D}$ configuration space.

Assuming the linear approximation for the beam dynamics through the machine lattice, the equation for the betatron motion of the beam centroid is

$$
\frac{d^{2}\left\langle y_{k}\right\rangle}{d s^{2}}+\kappa_{y}(s)\left\langle y_{k}\right\rangle=\frac{q}{E_{0}} \sum_{i=1} \delta\left(s-s_{i}\right) \Delta s_{i} \int d x d y d z E_{y}\left(x, y, s_{i} ; k, z\right) \rho_{k}(x, y, z) .
$$

In full generality one can think of the RHS of the above equation as a complicated function of the moments of the beam density. Notice that the dependence on the moments of the beam density comes not only from the direct term $\rho_{k}(x, y, z)$ but also indirectly through the electric field $E_{y}$ from its response to the passage of the $k-$ bunch. In fact, the dependence is more complicated as the electric field $E_{y}$ responds to the passage of previous beam bunches as well. A simple scenario is one in which the RHS of Eq. (5) is dominated by the displacement of the bunch centroids:

$$
\frac{q}{E_{0}} \int d x d y d z E_{y}\left(x, y, s_{i} ; k, z\right) \rho_{k}(x, y, z) \simeq \sum_{j=1}^{k} C_{k j}^{(i)}\left\langle y_{j}\right\rangle
$$

\footnotetext{
*mventurini@lbl.gov
} 
(The above equality defines the coupling coefficients $C_{k j}^{(i)}$.) The sum on the RHS term is restricted to $k$ by causality (having assumed that previous passages of the bunch train have had no impact on the current distribution of accumulated electrons; $k=1$ denotes the first bunch in the train.)

Ideally, in a machine experiment aimed at measuring EC induced tuneshifts one would like to excite betatron oscillations of the centroid by kicking a bunch in the train individually. The centroid amplitude of the kicked bunch will then evolve according to

$$
\frac{d^{2}\left\langle y_{k}\right\rangle}{d s^{2}}+\kappa_{y}(s)\left\langle y_{k}\right\rangle=\left\langle y_{k}\right\rangle C_{k k}^{(i)}
$$

with

$$
C_{k k}^{(i)}=\frac{q}{E_{0}\left\langle y_{k}\right\rangle} \int d x d y d z E_{y}\left(x, y, s_{i} ; k, z\right) \rho_{k}(x, y, z)
$$

where it is understood that all the bunches preceding bunch $k$, which have contributed to the establishment of the electric field $E_{y}\left(x, y, s_{i} ; k, z\right)$, traversed the EC station on-axis. To ease notation in the two equations above we have assumed that only one EC station is present, located at $s=s_{i}$

Using the familiar formula, the tuneshift experienced by the bunch centroid is then

$$
\Delta \nu_{y}(k)=\frac{1}{4 \pi} \beta_{y}\left(s_{i}\right) \Delta \kappa_{y}\left(s_{i}\right) \Delta s
$$

where $\Delta \kappa_{y}\left(s_{i}\right)=-C_{k k}^{(i)}$. The total tuneshift will be obtained by summing over all the EC stations.

In experimental situations where, as in Cesr, the time resolution of the kicker does not discriminate between individual bunches (all bunches in the train receiving the same kick), we argue that the tuneshift experienced by bunch $k$ will still be given by (8), with $C_{k k}^{(i)}$ given by (7), provided that the electric field in (7) be determined with all the leading bunches having the same vertical offset. This reflects the observation that if the coherent tuneshifts are sufficiently small and the measurement time sufficiently short all the bunches in a train will cross the EC station with about the same offset.

\section{MODELLING OF APRIL 2007 COHERENT TUNESHIFT MEASUREMENTS AT CESR}

In this section we report POSINST numerical simulations of the coherent tuneshift measurements made at Cesr in April 2007.

The 2007 experiments employed trains of 10 bunches separated by 14 ns with an additional 'witness' bunch following at a controlled distance. In the following we will refer to the set of the first 10 bunches as the bunch train 'proper'. The tuneshift measurements were carried out using BPM pick-ups after the bunches were kicked either horizontally or vertically (for horizontal and vertical tuneshift measurements respectively) with all the bunches experiencing the same kick amplitude. Measurements were carried out for both electron and positron beams but in this Report we will only focus on the simulations of the latter.

For a given offset $\Delta a=\Delta x, \Delta y$ of the bunches (including the witness bunch) we calculate the average electric field component experienced by the $k$-bunch in the direction $a=x, y$ of the bunch offset:

$$
\left\langle E_{a}(\Delta a)\right\rangle=\int d x d y d z E_{a}\left(x, y, s_{i} ; k, z\right) \rho_{k}(x, y, z)
$$

where $\rho_{k}(x, y, z)$, the bunch charge density (normalized to unity) is assumed to be in the from of a tri-gaussian density with rms widths $\sigma_{x}, \sigma_{y}$ and $\sigma_{z}$ and centroid offset $\Delta a \equiv\left\langle a_{k}\right\rangle=\int a \rho_{k}(x, y, z) d x d y d z$ in the $a$-direction. The electric field $E_{a}$ is calculated by POSINST [1]. Consistent with the considerations made at the end of Sec. I the electric field is determined with all the bunches in the train having the same offset. For this study we examined the EC build-up and the resulting electric field averaged over the positron bunch density in the Cesr drifts, and 'regular' dipoles.

Repeated runs are carried out to evaluate the average $\left\langle E_{a}\left(\Delta a_{j}\right)\right\rangle$ for various offsets $\Delta a_{j}$. In most cases we find that the dependence $\left\langle E_{a}\left(\Delta a_{j}\right)\right\rangle$ vs. $\Delta a_{j}$ is fairly linear and we fit the data against the linear function

$$
\left\langle E_{a}(\Delta a)\right\rangle=e_{a}^{(0)}+e_{a}^{(1)} \Delta a
$$

The e-cloud induced tuneshift contributed by the element of length $\Delta s$ is then estimated as 
TABLE I: Selected Parameters

\begin{tabular}{|l|c|c|}
\hline Description & & Value \\
\hline Energy & $E_{0}$ & $1.885 \mathrm{GeV}$ \\
Bunch population & $N$ & $1.2 \times 10^{10}$ \\
Bunch length & $\sigma_{z}$ & $12.59 \mathrm{~mm}$ \\
Avg. $\sigma_{x}$ in drifts & & $2.2 \mathrm{~mm}$ \\
Avg. $\sigma_{y}$ in drifts & & $0.16 \mathrm{~mm}$ \\
Avg. $\sigma_{x}$ in regular dipoles & & $2.2 \mathrm{~mm}$ \\
Avg. $\sigma_{y}$ in regular dipoles & $C$ & $0.14 \mathrm{~mm}$ \\
Ring circumference & & $174.86 \mathrm{~m}$ \\
Total length occupied by drifts & & $37.99 \mathrm{~m}$ \\
Total length occupied by regular dipoles & & $52.58 \mathrm{~m}$ \\
Total length occupied by soft dipoles & & $25.89 \mathrm{~m}$ \\
Total length occupied by hard dipoles & & $19.64 \mathrm{~m}$ \\
Avg. $\beta_{x}$ in drifts & & $18.82 \mathrm{~m}$ \\
Avg. $\beta_{y}$ in drifts & & $15.90 \mathrm{~m}$ \\
Avg. $\beta_{x}$ in regular dipoles & & $18.94 \mathrm{~m}$ \\
Avg. $\beta_{y}$ in regular dipoles & & 2 \\
Secondary Electron Yield (Al) & & 0.15 \\
Photon reflectivity in drifts & & 0.15 \\
Photon reflectivity in regular dipoles & & \\
\hline
\end{tabular}

$$
\Delta \nu_{a}^{(1)}=-\frac{e}{4 \pi E_{0}} \beta_{a} e_{a}^{(1)} \Delta s
$$

where $e>0$ is the positron charge.

In the case of regular dipoles with vertical beam offset we found an apparent deviation from linearity at large $\Delta a$ and we fit the data using the third-order polynomial

$$
\left\langle E_{a}(\Delta a)\right\rangle=e_{a}^{(0)}+e_{a}^{(1)} \Delta a+e_{a}^{(3)}(\Delta a)^{3}
$$

The cubic term in (12) contributes a nonlinear correction to the tune in large-amplitude betatron oscillations. Using perturbation theory and assuming that $\Delta a$, the amplitude of the offset, is much larger than the bunch rms transverse width we can estimate the nonlinear correction [3] as

$$
\Delta \nu_{a}^{(3)}=-\frac{3 e}{16 \pi E_{0}} \beta_{a} e_{a}^{(3)} \Delta s\langle a\rangle_{\max }^{2}
$$

where $\langle a\rangle_{\max }$ is the max. amplitude excursion experienced by the bunch centroid during betatron motion in the $a$ direction.

Results of the POSINST simulations are shown in figures 1-13. For each lattice element (regular dipole, drift) we consider ten different values of beam offsets in the horizontal and vertical plane and report:

1. The 3D field averages $\left\langle E_{a}\right\rangle$, in the direction $a=x, y$ along the bunch train (including the witness bunch);

2. The 3D field averages $\left\langle E_{a}\left(\Delta a_{i}\right)\right\rangle$ vs. the offset $\Delta a_{i}$ (and fitting polynomial) for the 10 bunches of the bunch train proper (i.e excluding the witness bunch).

3. The field gradient $e_{a}^{(1)}$ defined the as the linear coefficient of the polynomial fitting $\left\langle E_{a}\left(\Delta a_{i}\right)\right\rangle$ vs. $\Delta a_{i}$ and the corresponding contribution to the tuneshift (integrated over the machine length occupied by the lattice element). The error bars correspond to $3 \times$ the standard deviation error estimated from the fit.

In the case of regular dipoles with vertical beam offset, in addition to the linear tuneshift $\Delta \nu_{y}^{(1)}$ in (Fig. 6) we also report the estimated tuneshift including the nonlinear correction $\Delta \nu_{y}^{(3)}$, having assumed that the max. amplitude of the centroid oscillations is $4.5 \mathrm{~mm}$, see Fig. 7. Typical oscillation amplitudes of the beam centroids in the experiments were about $5 \mathrm{~mm}[2]$. The nonlinear correction is seen to be of the order of $10 \%$ and tends to reduce the EC-induced 
tuneshift. From comparison of Fig. 6 and 7 it is interesting to notice that the magnitude of the 'dip' in the value of tuneshift experienced by the 12th bunch, also observed in the measurements, depends somewhat on the nonlinearities and is more pronounced in linear approximation. Also, notice that this dip only appears in simulations of a dipole and not in those of a drift. It would be interesting to compare two different sets of measurements with different kick amplitudes to see if the nonlinear effects predicted by the simulations could be reproduced.

Finally, in Fig. 14 we add the contributions to the tuneshift from regular dipoles and drift and attempt a comparison with the actual measurements. The contribution from the regular dipoles was multiplied by a scaling factor meant to include an estimate of the contribution from the two other kinds of bends present in Cesr i.e. the 'hard' bends ( $B=0.198 \mathrm{~T}$ field, $25.9 \mathrm{~m}$ total length) and the 'soft' bends ( $B=0.107$ T field, $52.6 \mathrm{~m}$ total length). The scaling factor is based exclusively on the length of the machine portions occupied by the various bends: $(378+25.9+52.6) / 378 \simeq 1.21$. We should mention, however, that in our simulations for the regular dipoles a critical parameter, i.e. the number of photons per unit length per positron deposited on the interior wall of the vacuum chamber pipe per each bunch passage (parameter 'photpbppm' in POSINST), was already estimated as a weighted average of the presumed values in each kind of bends (the value used was 0.5316 no. of phot. $/ \mathrm{m} /$ positron vs. 0.4524 no. of phot. $/ \mathrm{m} /$ positron, the value pertaining to the actual regular dipoles).

Comparison between simulations and measurements in the vertical plane shows very close agreement. This, however, may be coincidental as the simulations do not include the contribution from the sections of the machine containing elements other than drifts and bends (i.e. portions occupied by quadrupoles, sextupoles, octupoles, wigglers, and solenoid). If these elements behaved like drifts the simulated tuneshits should be magnified by about one third (as the total length of occupied by these other elements, $115 \mathrm{~m}$, is more than half the total length of the drift spaces and the latter contribute about half the total tuneshift). This suggests that the value of some of the parameters used in the simulations, like the presumed electron secondary yield or the photon reflectivity, may have to be revised.

In simulations not reported here we studied how the electric field experienced by the bunches and the resulting tuneshifts change when one relaxes the assumption that the same offset should be attributed to all the bunches in the train. In most of the cases we examined the electric field experienced by each individual bunch did not appear to depend significantly on the offset of the preceding bunches with the exception of the case of horizontal offsets in regular dipoles. In this case we found that the field gradients experienced by a displaced bunch when the leading bunches in the train were on axis was significantly larger (than the case where the leading bunches had all the same offset as the trailing bunch).

Selected parameters relative to the machine setting during the measurements and POSINST simulations are reported in Table I. The complete POSINST input files used in the simulations are reported in the Appendices.

\section{ACKNOWLEDGMENTS}

This work was carried out with essential and much appreciated assistance from G. Dugan and J. Crittenden (Cornell. U), and M. Furman (LBNL). Work supported by the Department of Energy Contract no. DE-AC02-05CH11231.

\section{APPENDIX A: POSINST INPUT FILE FOR E-CLOUD CALCULATIONS IN REGULAR DIPOLES}

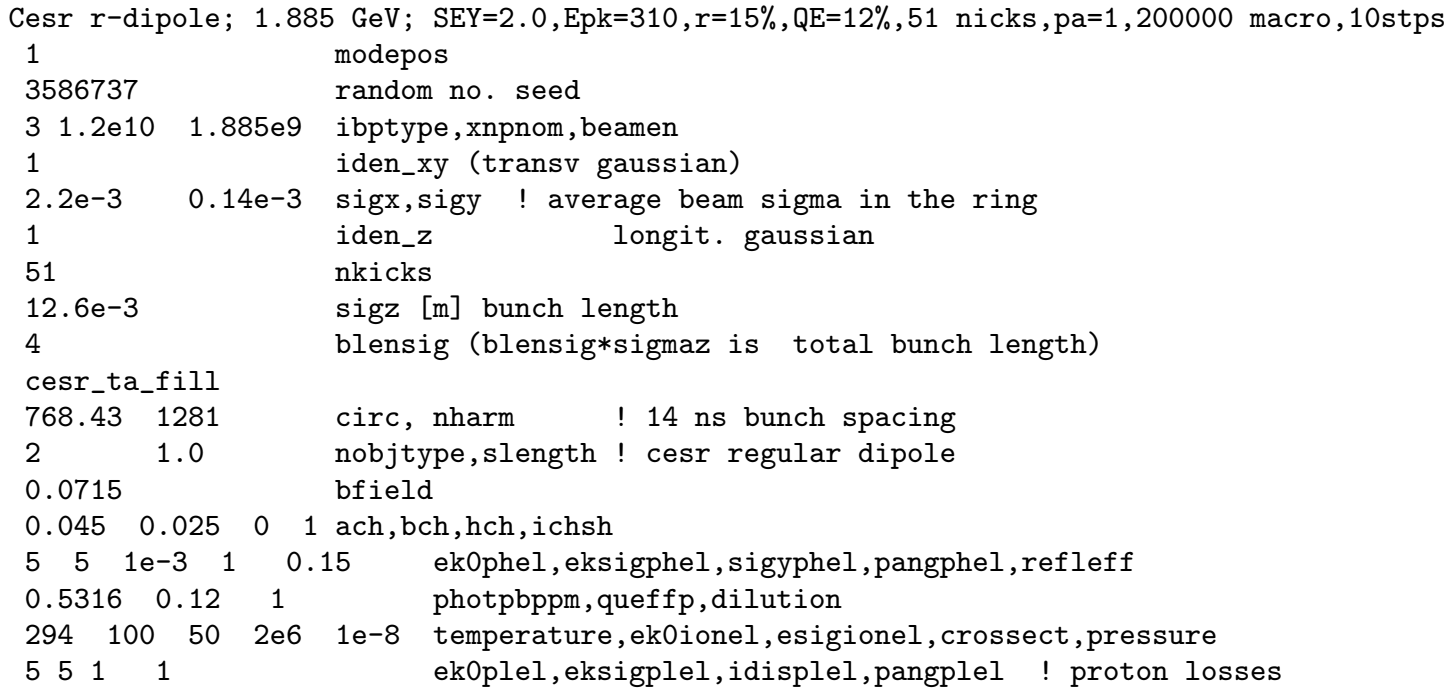




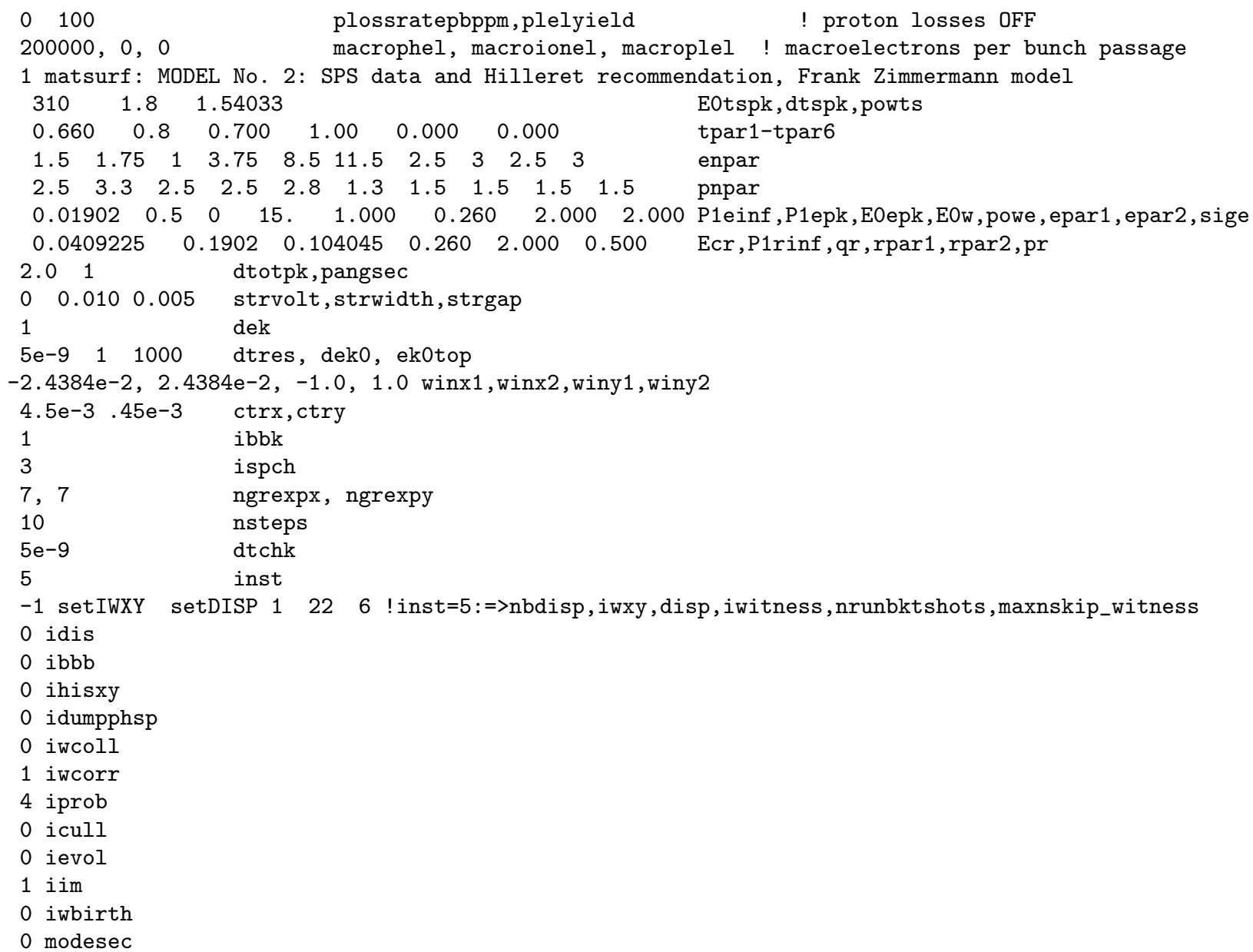

\section{APPENDIX B: POSINST INPUT FILE FOR E-CLOUD CALCULATIONS IN DRIFTS}

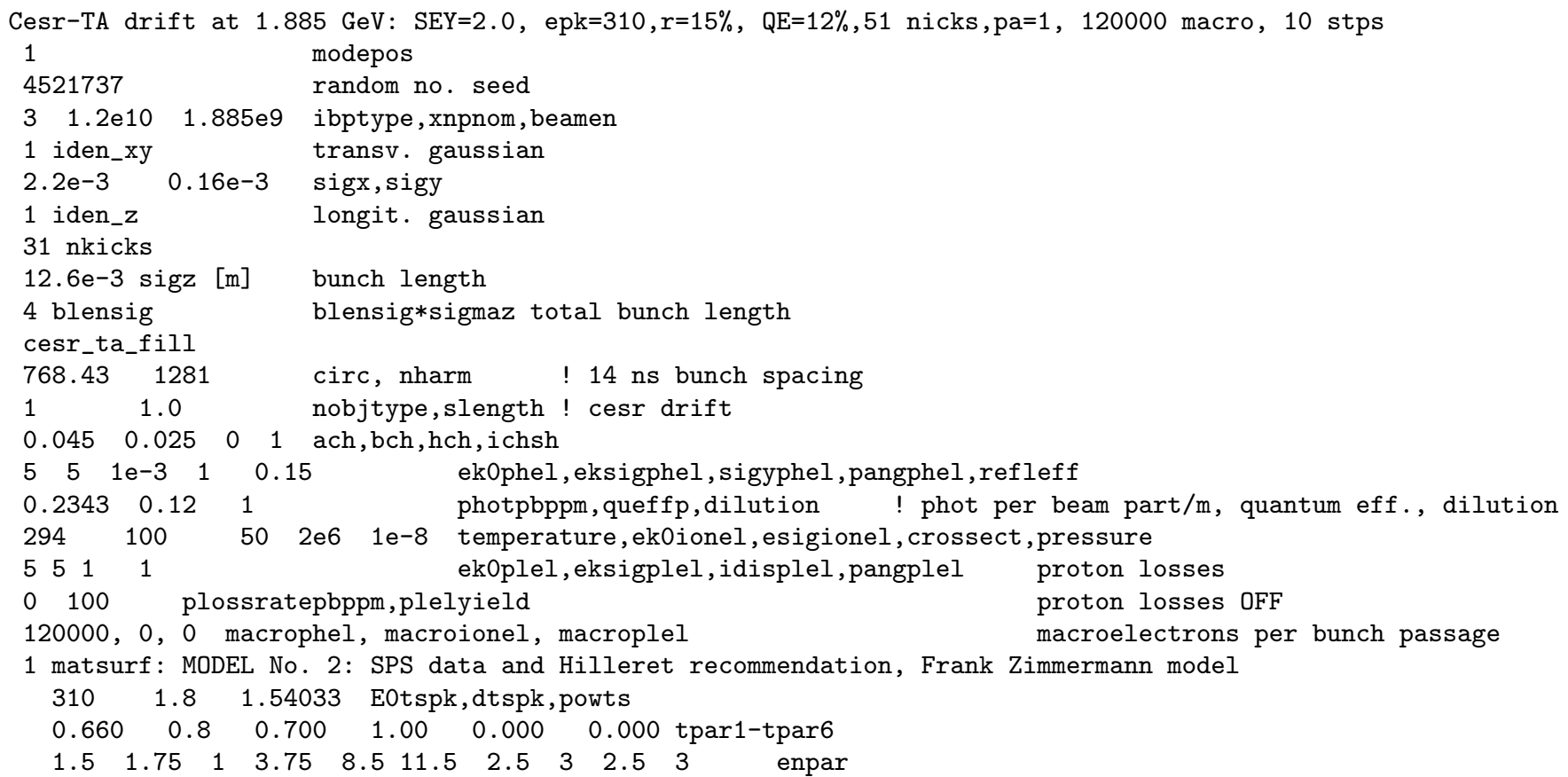


$\begin{array}{lllllllllll}2.5 & 3.3 & 2.5 & 2.5 & 2.8 & 1.3 & 1.5 & 1.5 & 1.5 & 1.5 & \text { pnpar }\end{array}$

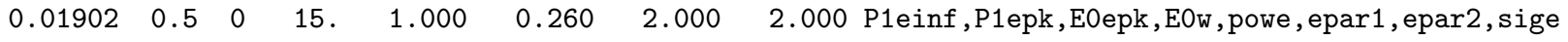

$0.0409225 \quad 0.1902 \quad 0.104045 \quad 0.260 \quad 2.000 \quad 0.500$ Ecr, P1rinf, qr, rpar1,rpar2,pr

2.01

$0 \quad 0.010 \quad 0.005$ strvolt, strwidth, strgap

1 dek

5e-9 11000 dtres, dek0, ek0top

$-2.4384 \mathrm{e}-2,2.4384 \mathrm{e}-2,-1.0,1.0$ winx 1 , winx 2 , winy 1 , winy2

$13.2 \mathrm{e}-3 \quad .96 \mathrm{e}-3 \quad \operatorname{ctrx}, \operatorname{ctry}$

1 ibbk

3 ispch

7,7 ngrexpx, ngrexpy

10 nsteps

$5 e-9$ dtchk

5 ! inst

-1 setIWXY setDISP 0216 ! inst=5:=> nbdisp, iwxy, disp, iwitness, nrunbktshots, maxnskip_witness

0 idis

$0 \mathrm{ibbb}$

0 ihisxy

0 idumpphsp

0 iwcoll

0 iwcorr

4 iprob

0 icull

0 ievol

1 iim

0 iwbirth

0 modesec

[1] M. A. Furman and M. T. F. Pivi, LBNL-49771, Phys Rev. ST-AB 5124404 (2003); and LBNL-52807.

[2] Robert Holtzapple, private communication.

[3] Start from a single-particle model $\frac{d^{2} y}{d s^{2}}+\kappa_{y}(s) y=\frac{q}{E_{0}} E_{y}$, where the electric field is represented by the third order polynomial $E_{0}=e_{y}^{(1)} y+e_{y}^{(3)} y^{3}$. Upon taking averages $\frac{d^{2}\langle y\rangle}{d s^{2}}+\kappa_{y}(s)\langle y\rangle=\frac{q}{E_{0}}\left(e_{y}^{(1)}\langle y\rangle+e_{y}^{(3)}\left\langle y^{3}\right\rangle\right)$. If the beam width is narrow compared to the beam offset we have $\left\langle y^{3}\right\rangle \simeq\langle y\rangle^{3}$. Next, we apply the canonical perturbation theory. In terms of the action angle variables $(J, \phi)$, with $y=\sqrt{2 \beta_{y} J} \cos \phi$, the Hamiltonian for this system reads $H=J / \beta_{y}(s)-\frac{q}{E_{0}}\left(e_{y}^{(1)} \beta_{y}(s) J \cos \phi+e_{y}^{(3)} \beta_{y}^{2}(s) J^{2} \cos ^{4} \phi\right)$. The tune (with linear and nonlinear corrections) is then obtained from $\frac{\partial H}{\partial J} / 2 \pi$ upon integrating over $s$ and averaging over the angle $\phi$. 

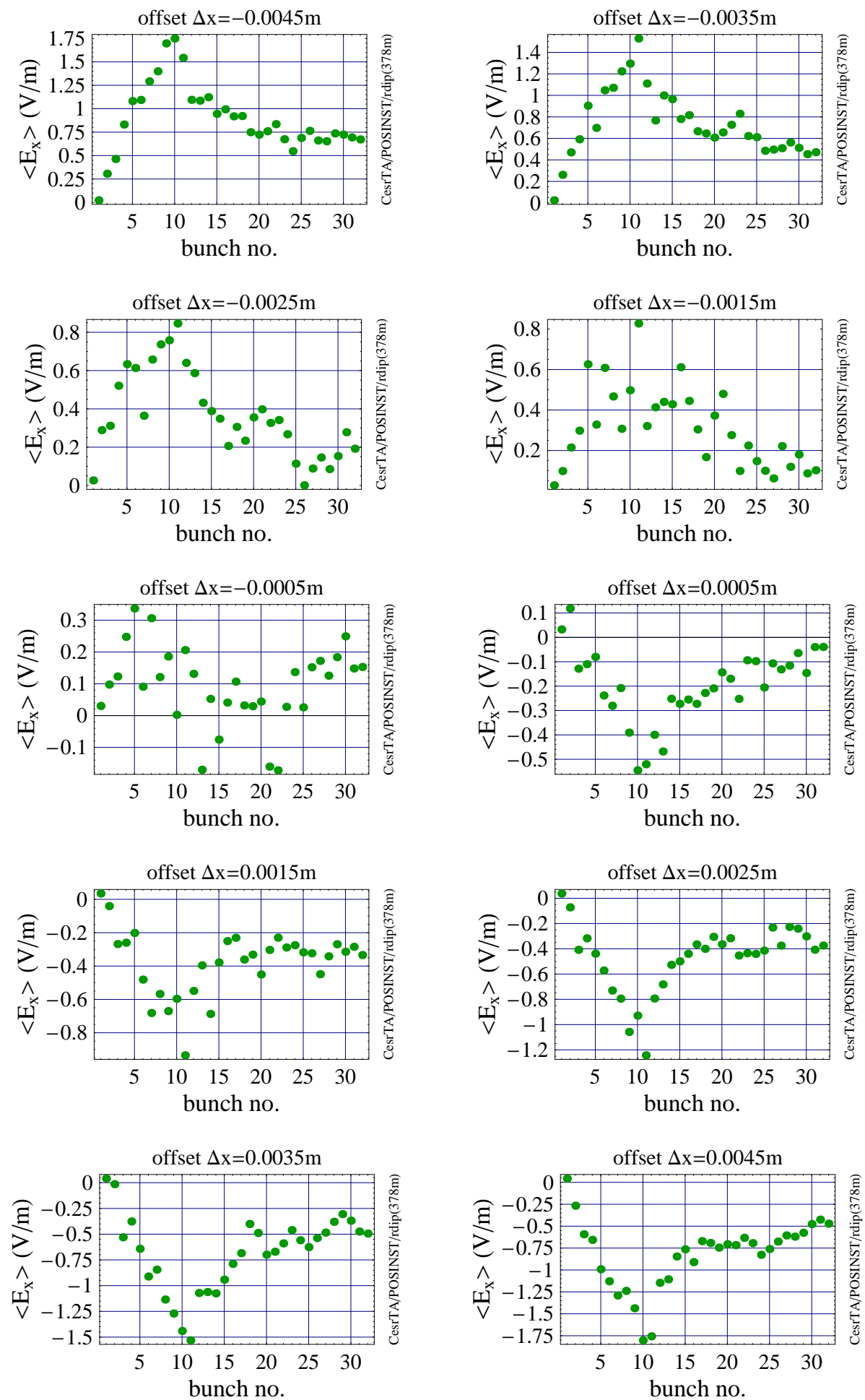

FIG. 1: Regular DIPOLE; horizontal offsets. Horizontal component of the electric field averaged over the bunch density along the bunch train for various horizontal offsets. The first 10 bunches belong to the bunch train proper. The other data points correspond to successive positions of a witness bunch. 

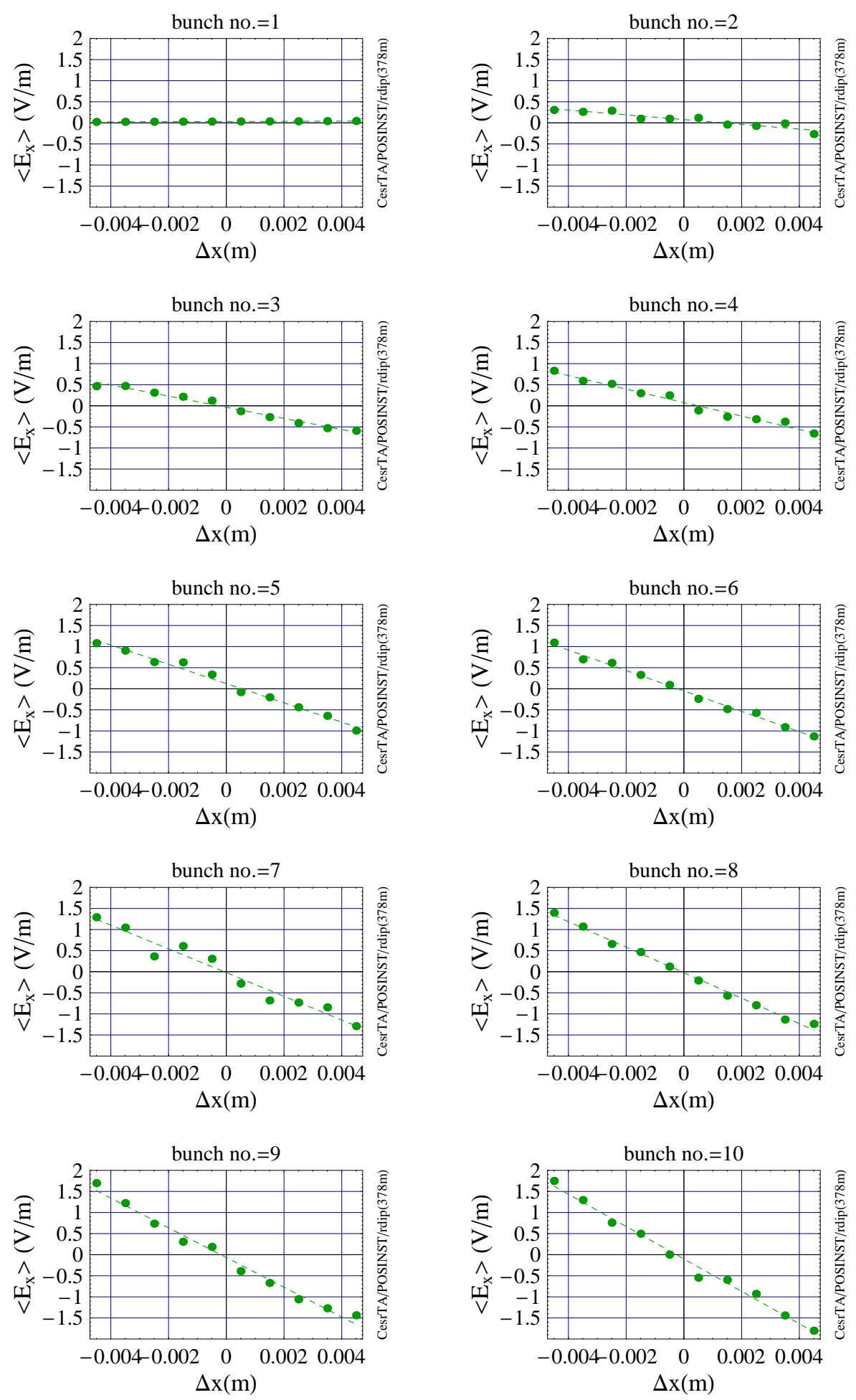

FIG. 2: Regular DIPOLE; horizontal offsets. Horizontal component of the electric field averaged over the bunch density vs. horizontal offsets for the 10 bunches in the train. 

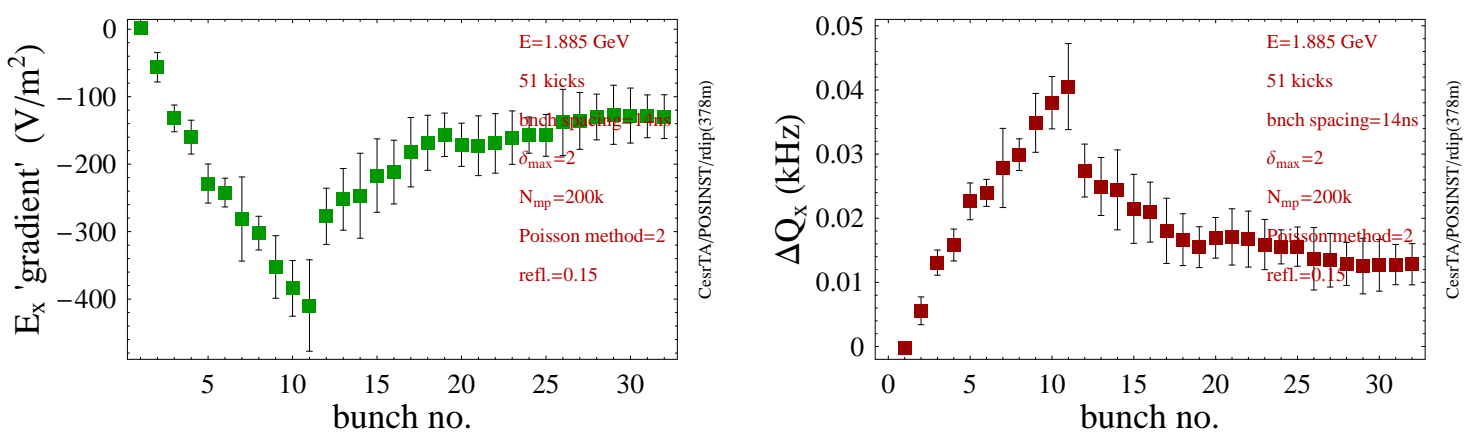

FIG. 3: Regular DIPOLE; horizontal offsets. Picture to the left: Horizontal field gradient $e_{x}^{(1)}$, see Eq. (10), along the bunch train computed from a linear fit of $\left\langle E_{x}\left(\Delta x_{i}\right)\right\rangle$ vs. $\Delta x_{i}, i=1,10$, (for the bunches in the train proper the data points used in the fit are those shown in Fig. 2). Picture to the right: contribution to coherent tuneshift resulting from all regular dipoles (total $377.99 \mathrm{~m}$ length). The error bars are $3 \times$ the standard deviation inferred for the linear fit. 

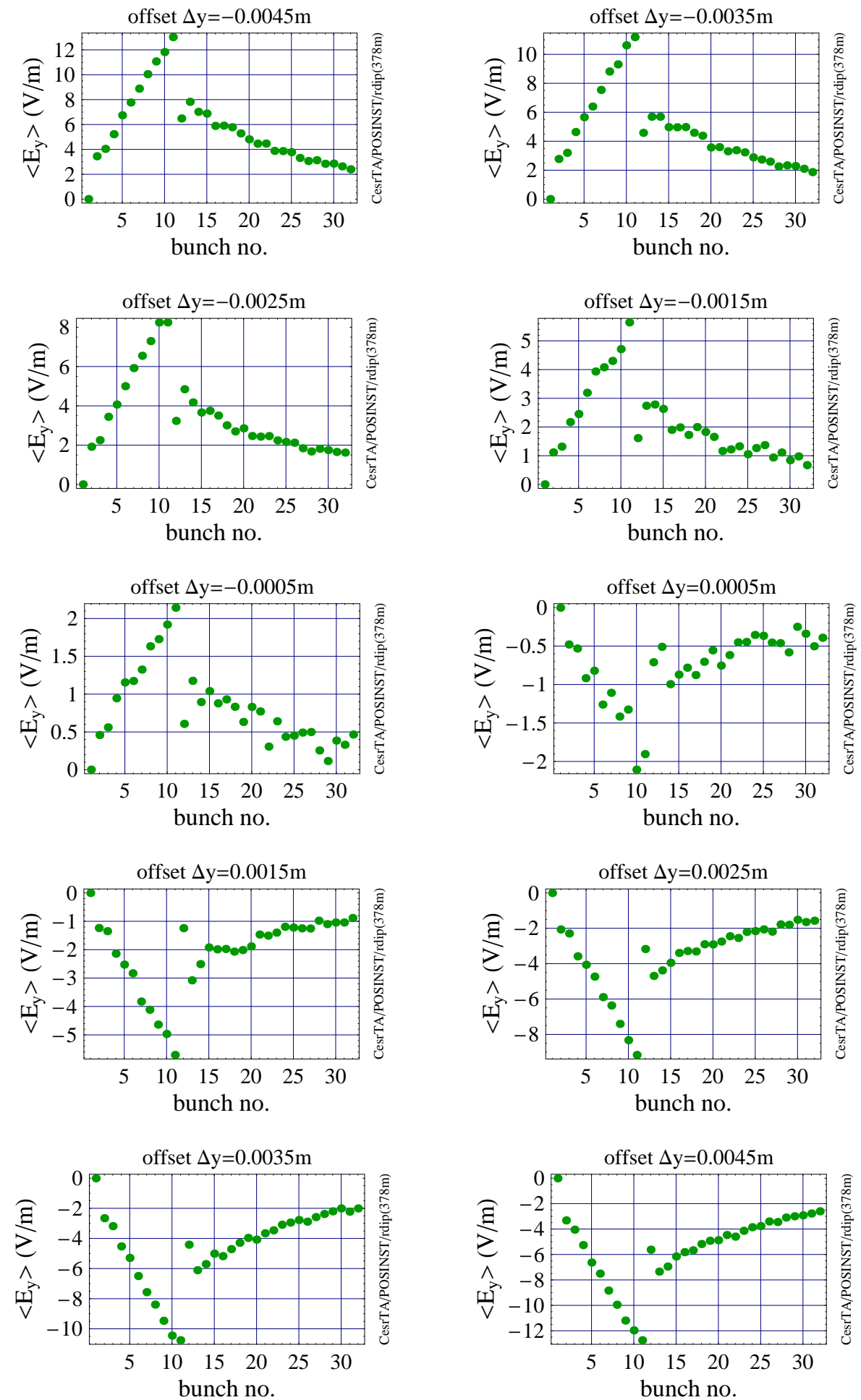

FIG. 4: Regular DIPOLE; vertical offsets. Vertical component of the electric field averaged over the bunch density along the bunch train for various horizontal offsets. The first 10 bunches belong to the bunch train proper. The other data points correspond to successive positions of a witness bunch. 

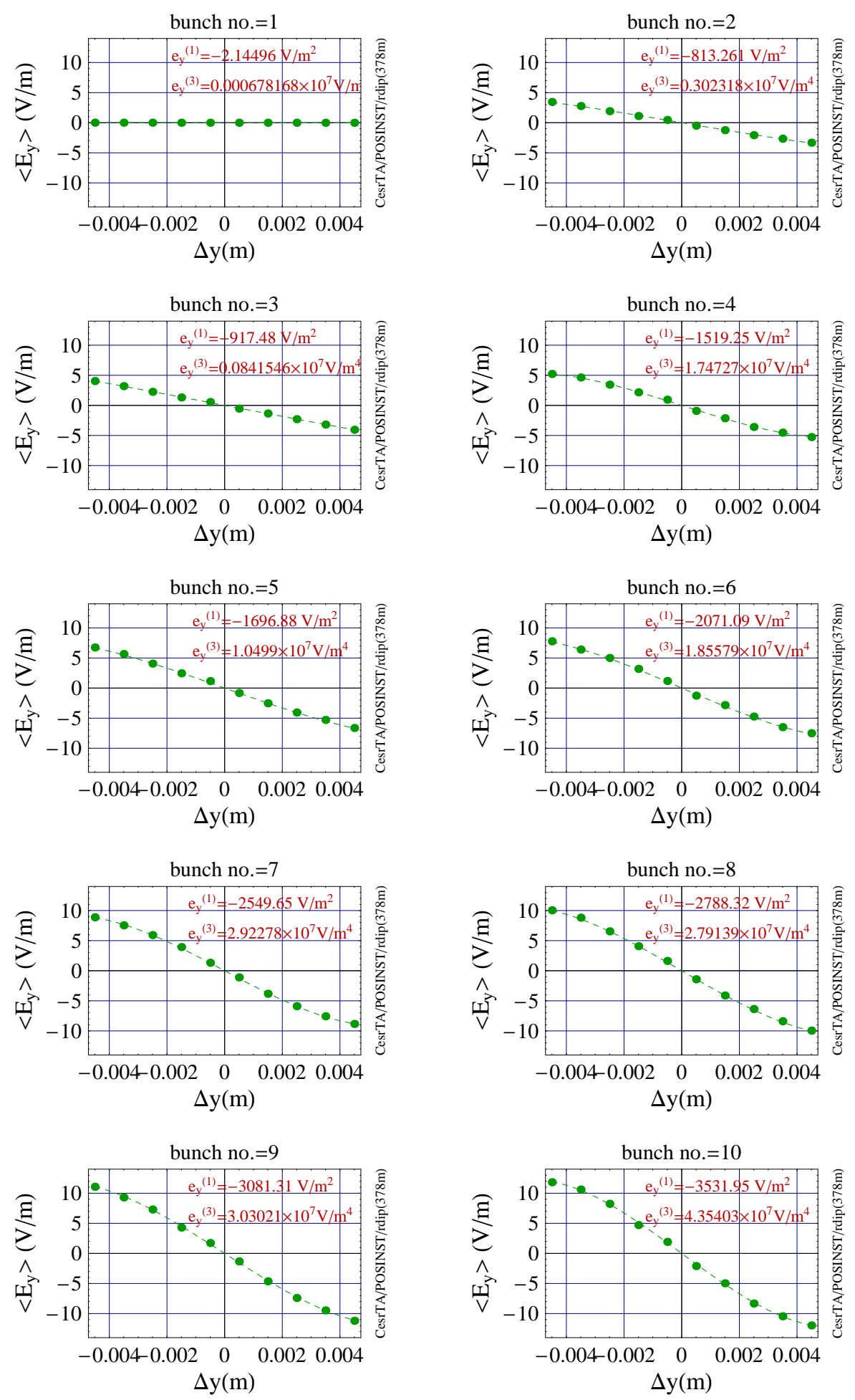

FIG. 5: Regular DIPOLE; vertical offsets. Vertical component of the electric field averaged over the bunch density vs. vertical offsets for the 10 bunches in the train. 

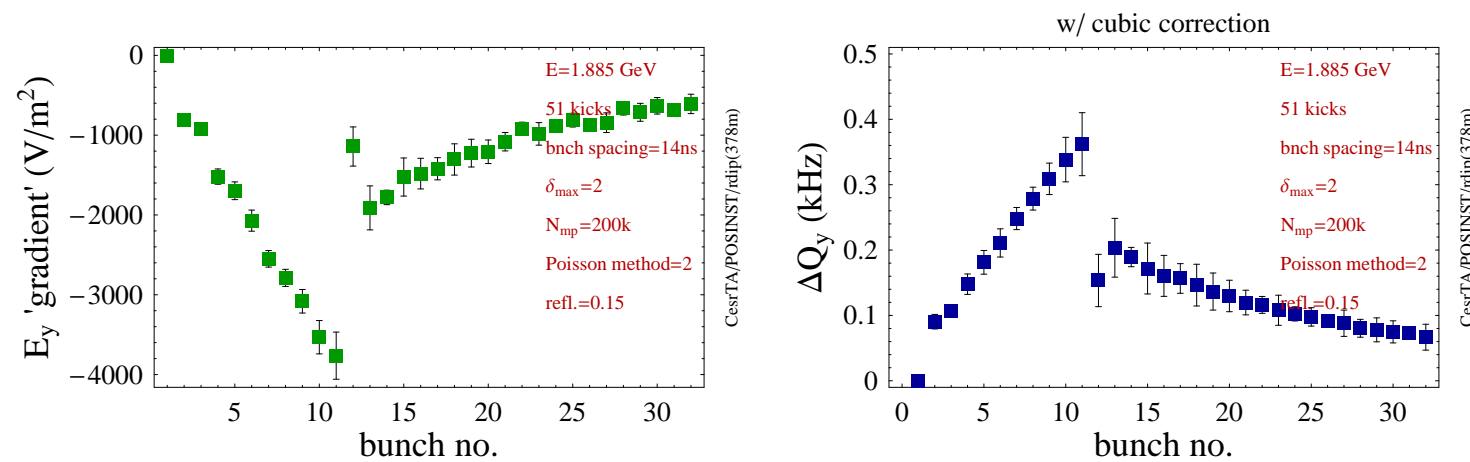

FIG. 6: Regular DIPOLE; vertical offsets. Picture to the left: Vertical field 'gradient' $e_{y}^{(1)}$, see Eq. (12), along the bunch train computed from fitting $\left\langle E_{y}\left(\Delta y_{i}\right)\right\rangle$ vs. $\Delta y_{i}, i=1,10$ against a polynomial with linear and cubic term (for the bunches in the train proper the data points used in the fit are those shown in Fig. 5). Picture to the right: contribution to coherent tuneshift resulting from all regular dipoles (total $377.99 \mathrm{~m}$ length) when both the linear and cubic terms are included having assumed a max. oscillation amplitude $\langle y\rangle_{\max }=4.5 \mathrm{~mm}$.

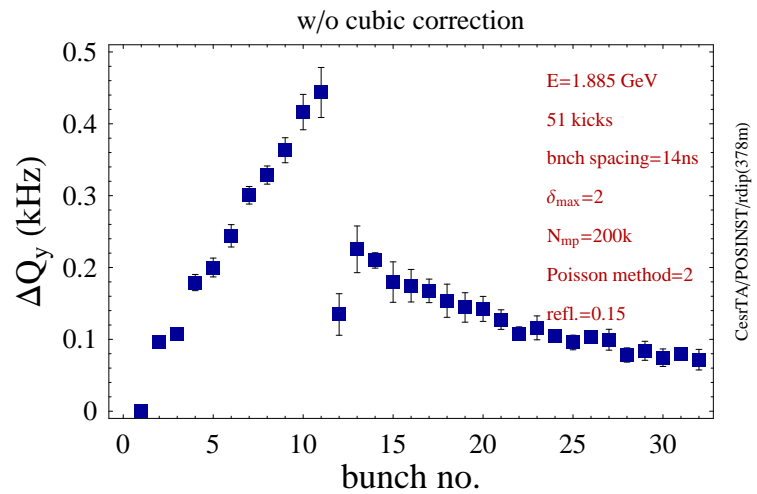

FIG. 7: Regular DIPOLE; vertical offsets. As in the right picture of Fig. 6 but with the difference that the tuneshift was estimated in the linear approximation. Notice that the relative magnitude of the 'dip' in the value of the estimated tuneshift experienced by the the 12th bunch is larger than in Fig. 6 . 

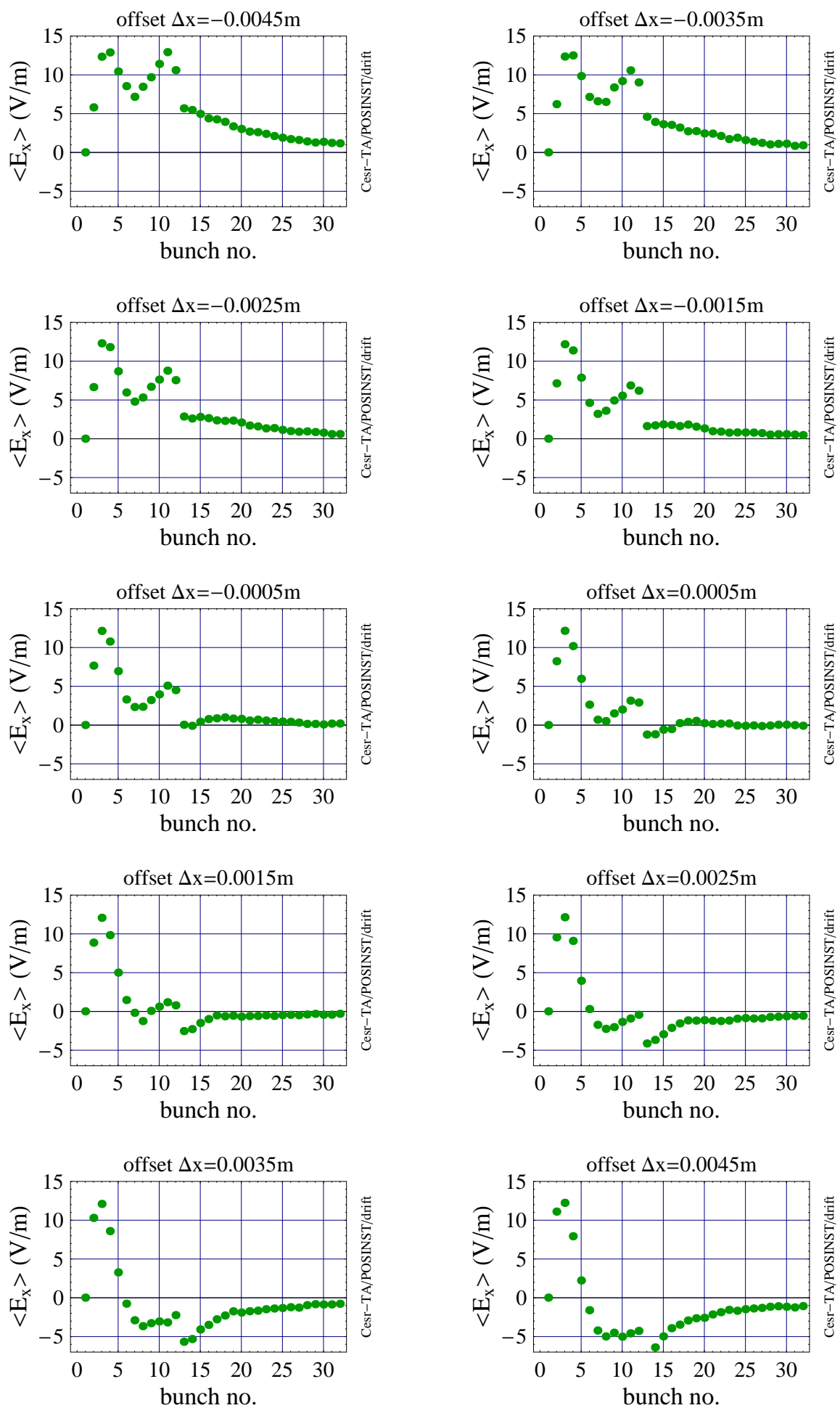

FIG. 8: DRIFT; horizontal offsets. Horizontal component of the electric field averaged over the bunch density along the bunch train for various horizontal offsets. The first 10 bunches belong to the bunch train proper. The other data points correspond to successive positions of a witness bunch. 

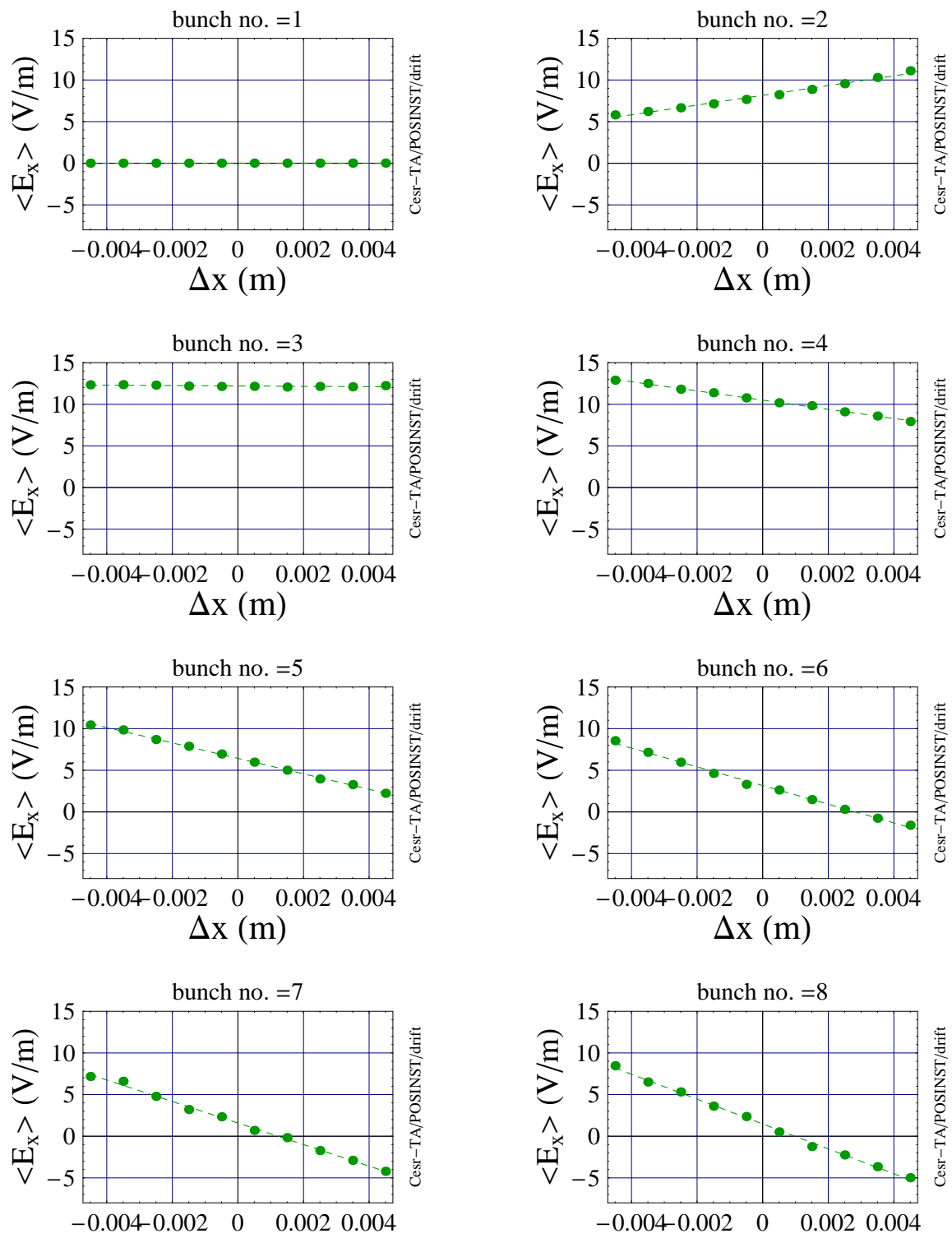

$\Delta \mathrm{x}(\mathrm{m})$

$\Delta \mathrm{x}(\mathrm{m})$
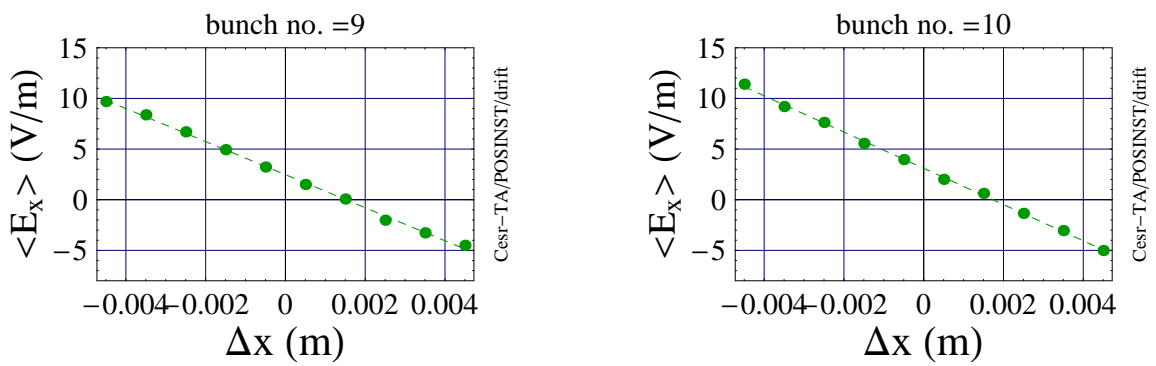

FIG. 9: DRIFT; horizontal offsets. Horizontal component of the electric field averaged over the bunch density vs. horizontal offsets for the 10 bunches in the train. 

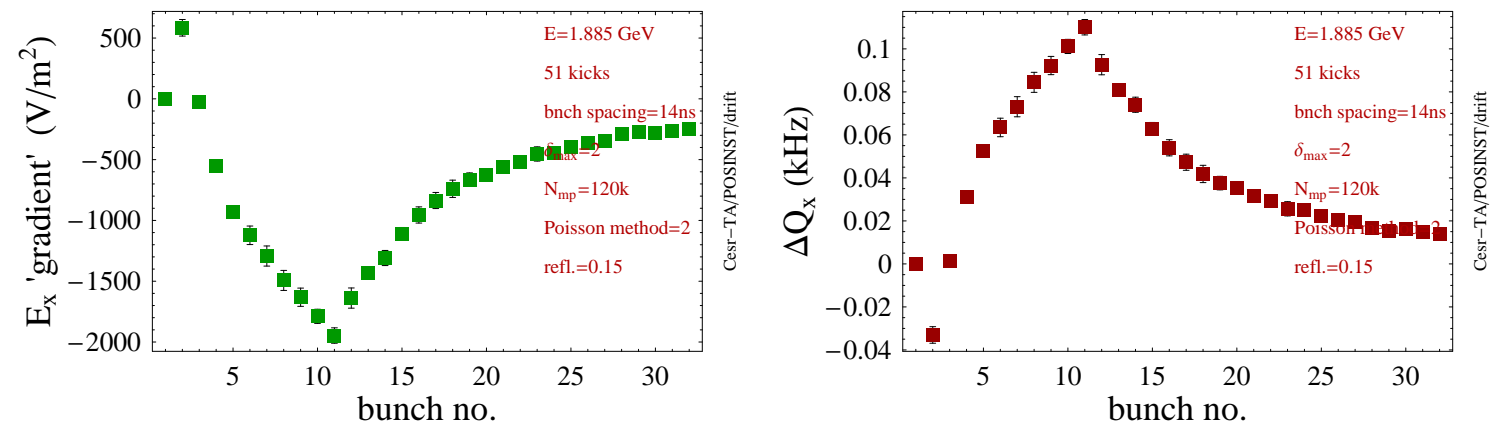

FIG. 10: DRIFT; horizontal offsets. Picture to the left: Horizontal field gradient $e_{x}^{(1)}$, see Eq. (10), along the bunch train computed from a linear fit of $\left\langle E_{x}\left(\Delta x_{i}\right)\right\rangle$ vs. $\Delta x_{i}, i=1,10$, (for the bunches in the train proper the data points used in the fit are those shown in Fig. 2). Picture to the right: contribution to coherent tuneshift resulting from all regular dipoles (total $174.8 \mathrm{~m}$ length). 

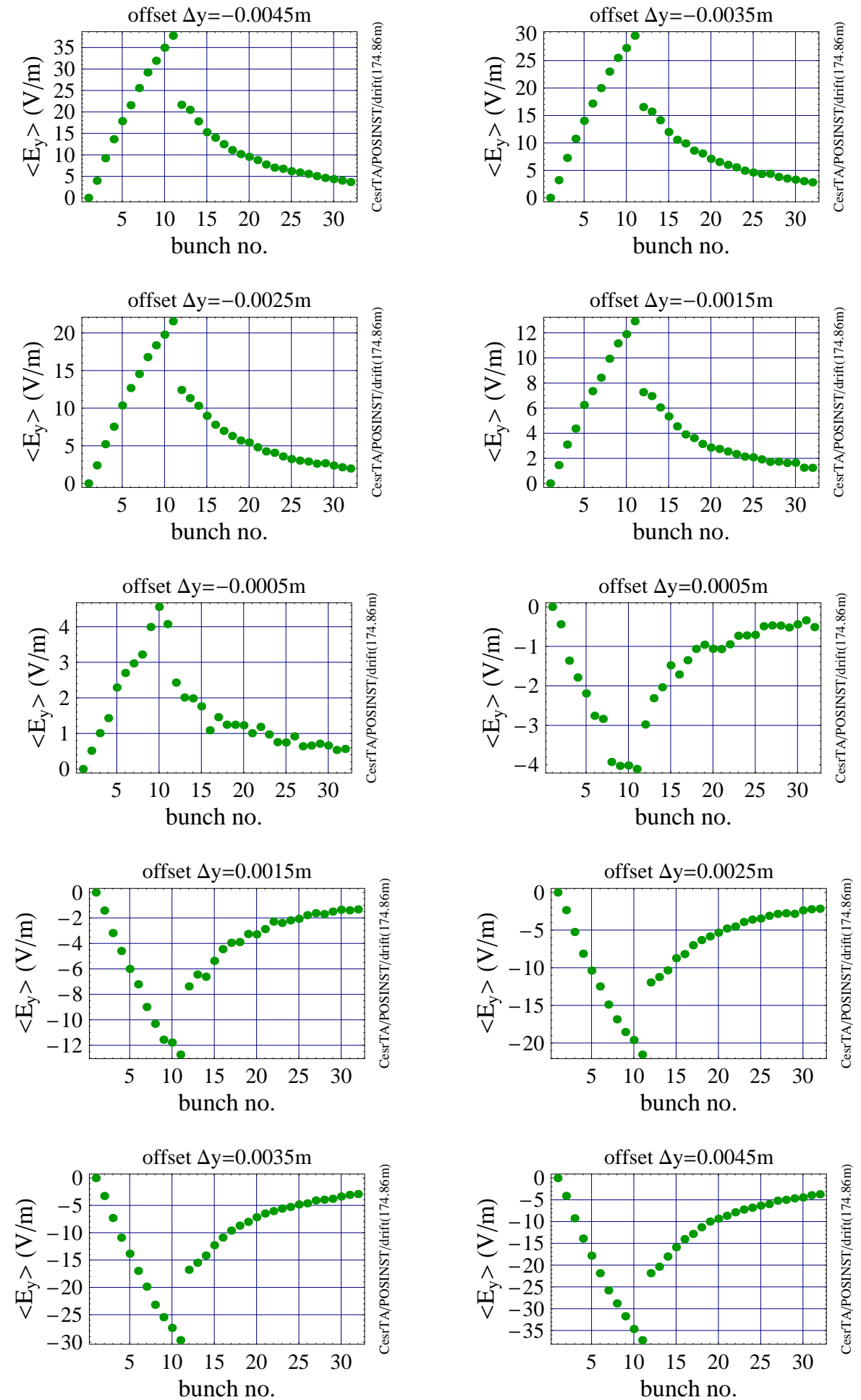

FIG. 11: DRIFT; vertical offsets. Vertical component of the electric field averaged over the bunch density along the bunch train for various horizontal offsets. The first 10 bunches belong to the bunch train proper. The other data points correspond to successive positions of a witness bunch. 

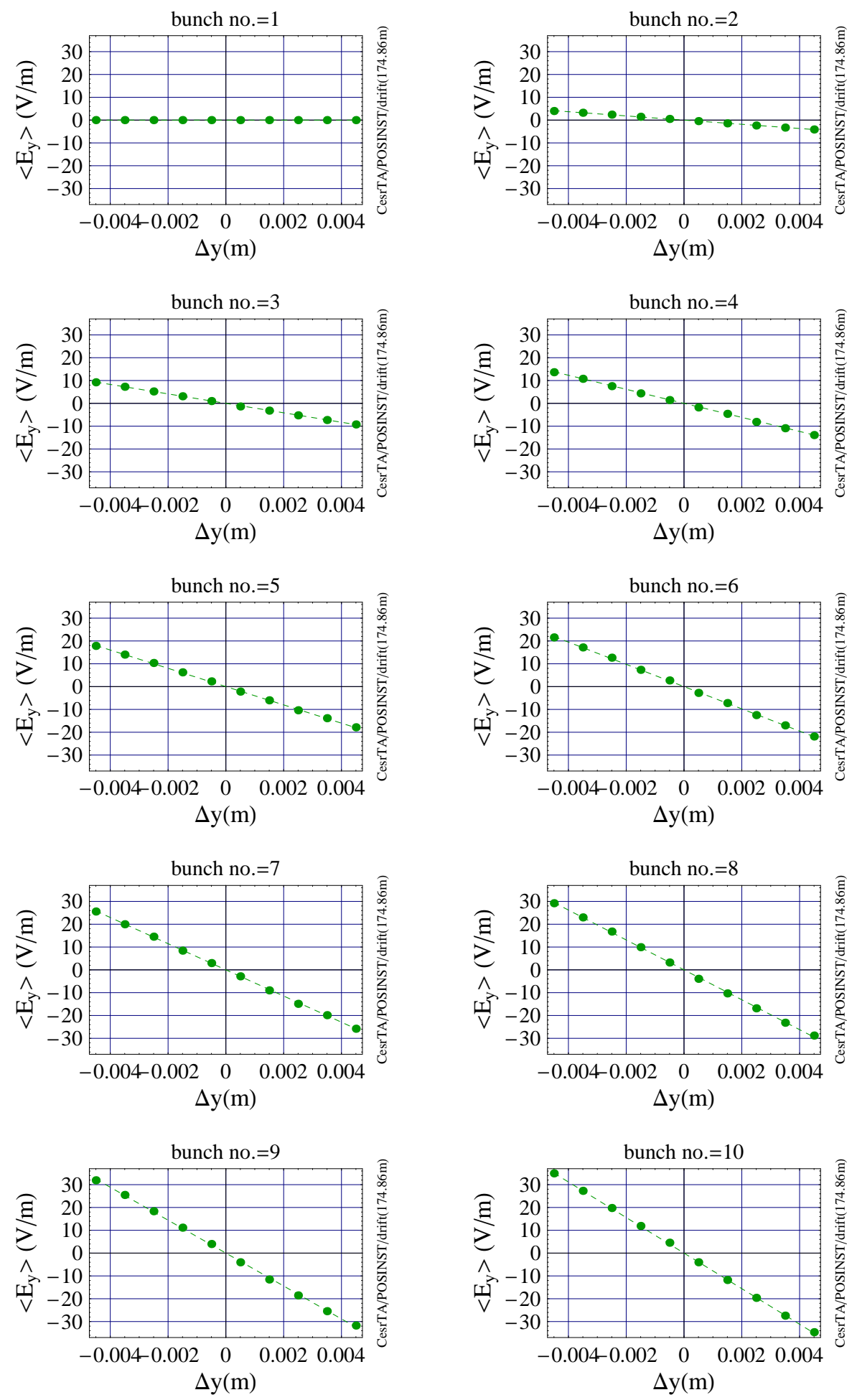

FIG. 12: DRIFT; vertical offsets. Vertical component of the electric field averaged over the bunch density vs. vertical offsets for the 10 bunches in the train. 

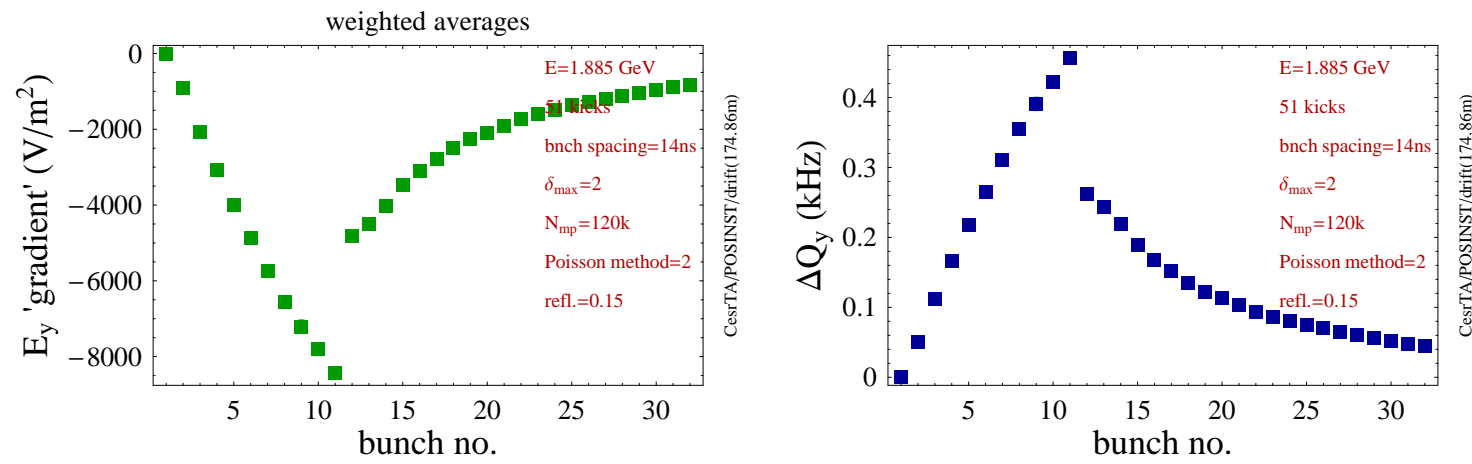

FIG. 13: DRIFT; vertical offsets. Picture to the left: Vertical field gradient $e_{y}^{(1)}$, see Eq. (10) along the bunch train computed from a linear fit of $\left\langle E_{y}\left(\Delta y_{i}\right)\right\rangle$ vs. $\Delta y_{i}, i=1,10$, (for the bunches in the train proper the data points used in the fit are those shown in Fig. 12). Picture to the right: contribution to coherent tuneshift resulting from all drift spaces (total $174.8 \mathrm{~m}$ length).
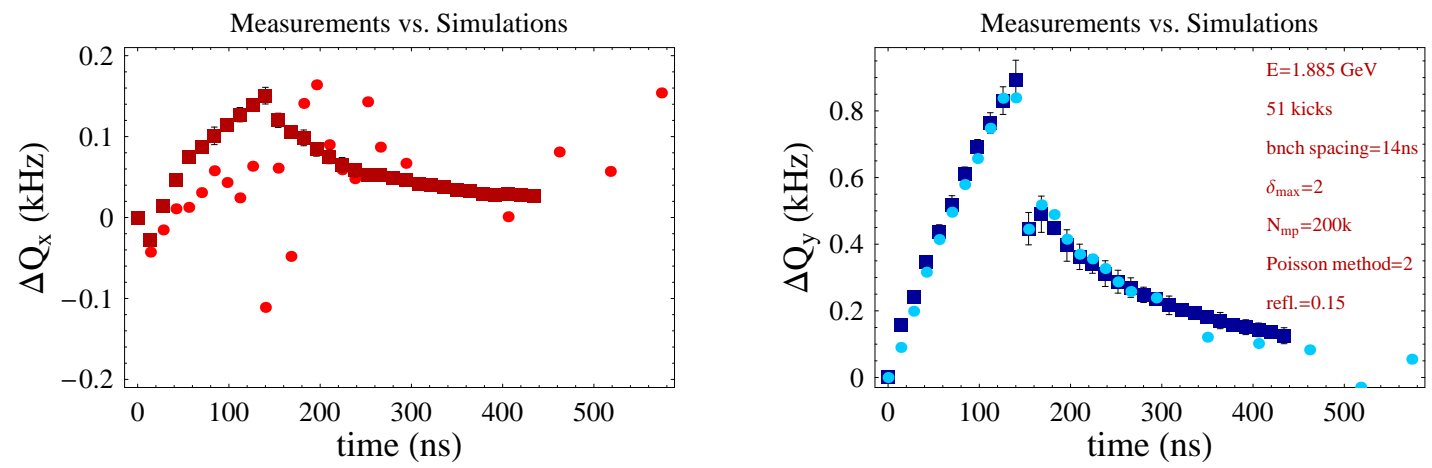

FIG. 14: The tuneshift contribution from all drift spaces and dipoles as simulated with POSINST and show in the previous pictures are added up (square data points) and compared with tuneshift measurements (dots) carried out at Cesr in 2007. The contribution from the regular dipoles is scaled to include an estimate of the contribution from the 'soft' and 'hard' bends as well. The simulated values of the tuneshifts do not include the contribution from sections of the machine occupied by wigglers $(24.55 \mathrm{~m})$, and other elements (quadrupoles, sextupoles, octupoles, solenoid, adding to a total $94.44 \mathrm{~m}$ length). 